\title{
Mundos que colapsan
}

\section{Collapsing worlds}

\author{
Alejandro Camargo \\ Universidad del Norte, Barranquilla. \\ $\square$ fcamargoa@uninorte.edu.co \\ (1) ORCID: 0000-0002-5812-8416 \\ ज् Google Scholar
}

Vivimos en un mundo que amenaza con colapsar de múltiples formas. El cambio climático, la intensificación de desastres ambientales a escala planetaria y la pandemia del Covid-19 son algunos de los principales fenómenos que, al amenazar nuestra existencia, nos recuerdan también la ambivalencia de las fuerzas vitales. Por un lado, se reafirma la idea del Antropoceno, una reificación del dominio de los humanos sobre la tierra que los ha convertido en una fuerza geológica. Esta idea ya ha sido suficientemente criticada por desdibujar las diferencias sociales (Biermann et al., 2016), subestimar el lugar del capitalismo y su historicidad en la naturaleza (Moore, 2017), y por estar marcada por enfoques masculinistas y tecnonormativos (Grusin, 2017). Aun así, existe un consenso sobre el origen antrópico de la crisis ambiental global actual. Por otro lado, la intensidad y frecuencia crecientes en la ocurrencia de desastres ambientales nos muestra que las transformaciones antrópicas del entorno pueden llevar a un escalamiento de fuerzas que se vuelcan sobre los humanos y que superan cualquier intento de control. De esta manera, la amenaza del colapso engendra una tensión entre múltiples fuerzas humanas y no humanas que dan forma a diversas posibilidades de vida en un mundo que cambia de forma abrupta. La vida, en otras palabras, es lo que está en juego ante el colapso del mundo.

El interés creciente por la vida en antropología y en las ciencias sociales en general es precisamente una respuesta a los fenómenos que ponen en riesgo la existencia en este momento histórico. Sin embargo, como plantea João de Pina-Cabral (2018), la idea de la vida siempre ha estado presente 
entre las herramientas analíticas de las ciencias sociales (véase también Maldonado, 2016). Consideremos, por ejemplo, el concepto vida cotidiana. Este concepto ha inspirado trabajos fundamentales en teoría social que se preguntan desde lo cotidiano por fenómenos más amplios como el capitalismo, la interacción social, la violencia y el consumo (véase, por ejemplo, Das, 2016; De Certeau, 1988; Goffman, 1981; Lefebvre, 1991). Para la antropología y la etnografía la vida cotidiana es, de hecho, uno de los escenarios primordiales para la descripción y el análisis. De igual manera, la antropología se ha interesado en formas de vida no humana, principalmente desde las cosmologías no occidentales, así como por el final de la vida: la muerte. ¿Entonces qué es lo nuevo en las inquietudes contemporáneas sobre la vida en tiempos de colapso, crisis y desastre?

El interés actual por la vida en antropología, las humanidades y las ciencias sociales en general (a veces en la intersección de esos campos) dan continuidad a esas preocupaciones, pero a su vez tiene varias particularidades. Una de ellas consiste en la atención a la vida más allá de lo humano. Si bien la antropología se ha interesado por los mundos de seres, animales y plantas, y la vida de montañas, ríos y otras geoformas, en el momento actual ha expandido su mirada, por ejemplo, al universo de lo microscópico, a la materialidad de esos y otros mundos, y a la intersección de conocimientos científicos y no científicos en torno a diversos seres y elementos (véase, por ejemplo, el número especial sobre Conflicto y paz en Colombia, más allá de lo humano en Maguaré vol. 33 n. ${ }^{\circ} 2$ de 2019 y Swanson, 2017). Una segunda particularidad sobre las preguntas por la vida en un mundo que colapsa, tiene que ver con la tensión entre la destrucción y la permanencia y el lugar de esa tensión en el capitalismo global. De allí el interés creciente por la extinción de especies (Sodikoff, 2011; Van Dooren, 2014) y por la vida en ambientes en ruina y destruidos por el capitalismo y la modernización (véase, por ejemplo, Kirksey, 2014; Tsing, 2015; Tsing et al. 2017). La vida es, por lo tanto, un ámbito de investigación interdisciplinar, una categoría de análisis social, y una preocupación política y social ante la crisis planetaria actual.

El colapso del mundo es en realidad una multiplicidad de experiencias heterogéneas, dentro de la cual se encuentra la degradación de la vida y los elementos del planeta. Pero el planeta no es el único mundo que colapsa. Por eso hablamos de forma más precisa sobre mundos en plural. La antropología de los desastres nos ha mostrado cómo lugares concretos se destruyen materialmente, y al hacerlo se derrumban los mundos construidos por el trabajo, el parentesco, y la comunidad. A su vez, el desmembramiento de esos mundos se conecta con crisis más amplias y de más larga duración. Pensemos, por ejemplo, en Puerto Rico. 
El huracán María en 2017 afectó de forma dramática la infraestructura y las formas de vida, pero también hizo más evidente la vulnerabilidad y la desigualdad histórica asociadas al colonialismo de los Estados Unidos y el desarrollo del capitalismo en la isla (Rivera, 2020; Rodríguez, 2020). Lo mismo podríamos decir en el caso de la destrucción generada por la guerra. La vida en zonas de conflicto donde la muerte es una realidad cercana y cotidiana es una experiencia emocional, sensorial y material del mundo que se desmorona en medio de la inequidad.

Pero el colapso del mundo no se experimenta solo durante eventos catastróficos de gran alcance. Hay otros mundos que se desvanecen lentamente entre lo material, lo emocional y lo espiritual de lo cotidiano. En este sentido, el concepto de solastacia ha surgido como una forma de explicar la aflicción emocional y existencial producto del deterioro ambiental y la pérdida de esos lugares de la naturaleza que son significativos socialmente (Albrecht et al., 2007). En el campo de lo espiritual, por su parte, hay quienes esperan el fin del mundo profesado desde sus creencias. Este momento esperado es en realidad una transición de un mundo que contradice los valores y significados espirituales a un mundo de perfección y vida eterna. En ocasiones esa espera se orienta a una fecha y hora específicas. Tal como sucedió en enero de 2021, cuando una pequeña comunidad religiosa en el caribe colombiano se alistó para esperar "el rapto" de Jesucristo, un momento en el que iban a abandonar el mundo actual en un viaje sin retorno. Como este, muchos otros grupos a lo largo de la historia y en diferentes lugares han esperado estos momentos de transición material y espiritual. Para estos grupos, el fin del mundo es un anhelo y no necesariamente una catástrofe. Lo catastrófico y perturbador sería permanecer en el mundo terrenal. Pero más allá del desenlace de estas historias (que en ocasiones ha sido dramático), nos queda preguntarnos por qué tantos grupos, diferentes entre sí, han deseado abandonar el mundo actual. Quizás la respuesta no tenga que ver solo con el plano espiritual y religioso de ideas que incluso se remontan siglos atrás. La respuesta puede tener que ver también con cómo lo espiritual y lo religioso resulta ser una vía (la única para muchos) para escapar de las desigualdades e injusticias que implican vivir en un sistema capitalista. En este sistema, millones de personas son condenadas a vivir en un mundo que colapsa entre el desempleo, las deudas, el hambre y la miseria.

Ni la fuerza destructora, ni la aquiescencia agotan las posibilidades de los humanos en un mundo que colapsa. Mientras algunas personas esperan por una transición en el plano espiritual y religioso, otras lo buscan en el material mediante luchas por un mundo más justo y equitativo. Diversos movimientos sociales y ambientales reafirman, diversifican 
y expanden sus luchas ante la destrucción de sus mundos y territorios. El extractivismo, la contaminación, el desecamiento de cuerpos de agua y la deforestación son algunos de los fenómenos devastadores que han encontrado un obstáculo en las comunidades que se organizan para frenarlos. Pero estas luchas ocurren a medida que la misma fuerza destructora del capitalismo se reconfigura para ofrecer una solución a sus propias contradicciones. Como plantea Lilley (2012), el capitalismo usa las crisis y las tragedias de la vida cotidiana para reproducirse y expandirse (p. 2). Este es el caso de propuestas desde el mercado como la economía verde, la geoingeniería, los mercados de carbono y las energías limpias. Estas propuestas aparecen como soluciones, pero al mismo tiempo el trabajo desde la antropología y otros campos ha demostrado que también engendran otras tensiones. Estas respuestas al colapso del mundo, paradójicamente crean las condiciones para erosionar otros mundos.

Este dossier propone una reflexión sobre los mundos que colapsan y en los que convergen diversas geografías, historias y subjetividades. María Isabel Galindo, cuenta cómo en el caso del Pacífico colombiano la vida de la gente costera ocurre entre el colapso y la creación impulsadas por las fuerzas del agua del mar. Estas fuerzas se entretejen, a su vez, con historias de violencia, de deterioro de la pesca y de desigualdad socioeconómica. Ariana Mendoza, presenta la historia de cómo la desecación de uno de los lagos que existían en lo que hoy es la Ciudad de México es experimentado como una pérdida dolorosa, una forma de despojo y como un desastre lento y progresivo. Mónica Cuéllar, utiliza una copla popular sobre el fin del mundo campesino en los Andes colombianos para reflexionar sobre historias de migración, deterioro ambiental, soledad y tristeza. Gladys Espinel, analiza las narraciones sobre la destrucción de un casco urbano ubicado en las faldas de los Andes colombianos como consecuencia de una remoción en masa asociada a una falla geológica y a lluvias torrenciales. Junto a la explicación científica, las narrativas sobre el fin del mundo en este lugar incluyen también historias mágicas y sagradas. En su conjunto, los cuatro textos proveen elementos analíticos, teóricos y empíricos para poder comprender de forma más amplia cómo y con qué fin diferentes sujetos narran el colapso del mundo, cuál es la textura material y ecológica de esos mundos y cómo se entrelaza esa textura con la experiencia vivida en ellos, qué nociones de la historia atraviesan esas narrativas sobre el colapso o el fin del mundo, qué nuevos mundos nacen del colapso y las ruinas de otros mundos y qué desafíos teóricos emergen de la exploración etnográfica y de archivo de estas narrativas, experiencias y materialidades. Por lo tanto, esperamos que este número especial sirva de inspiración para otras indagaciones y para contribuir a la antropología de los desastres, las catástrofes y el colapso. 


\section{Referencias bibliográficas}

Albrecht, G., Sartore, G. M., Connor, L., Higginbotham, N., Freeman, S., Kelly, B., ... y Pollard, G. (2007). Solastalgia: the distress caused by environmental change. Australasian psychiatry, 15(sup1), S95-S98.

Biermann, F., Bai, X., Bondre, N., Broadgate, W., Chen, C. T. A., Dube, O. P., ... y Seto, K. C. (2016). Down to earth: contextualizing the Anthropocene. Global Environmental Change, 39, 341-350.

Das, V. (2006). Life and Words: Violence and the Descent into the Ordinary. University of California Press.

De Certeau, M. (1988). The practice of everyday life. University of California Press.

Goffman, E. (1981). La presentación de la persona en la vida cotidiana. Amorrortu.

Grusin, R. (ed.). (2017). Anthropocene feminism. University of Minnesota Press.

Kirksey, E. (ed.). (2014). The multispecies salon. Duke University Press.

Lefebvre, H. (1991). Critique of everyday life. Verso.

Lilley, S. (2012). The apocalyptic politics of collapse and rebirth. En S. Lilley, D. McNally, E. Yuen \& J. Davis (eds.), Catastrophism: the apocalyptic politics of collapse and rebirth (pp. 1-14). PM Press.

Maldonado, C. E. (2016). Hacia una antropología de la vida: elementos para una comprensión de la complejidad de los sistemas vivos. Boletín de Antropología, 31(52), 285-301.

Moore, J. W. (2017). The Capitalocene, Part I: on the nature and origins of our ecological crisis. The Journal of peasant studies, 44(3), 594-630.

Pina-Cabral, J. D. (2018). Turning to life: A comment. HAU: Journal of Ethnographic Theory, $8(3), 522-529$.

Rivera, D. Z. (2020). Disaster Colonialism: A Commentary on Disasters beyond Singular Events to Structural Violence. International Journal of Urban and Regional Research. https://doi.org/10.1111/1468-2427.12950

Rodríguez, I. (2020). Mutual Aid and Survival as Resistance in Puerto Rico. NACLA Report on the Americas, 52(3), 303-308. https://www.tandfonline.com/doi/ abs/10.1080/10714839.2020.1809099?journalCode=rnac20

Sodikoff. G. M. (2011). The Anthropology of Extinction: Essays on Culture and Species Death. Indiana University Press.

Swanson, H. A. (2017). Methods for multispecies anthropology: thinking with salmon otoliths and scales. Social Analysis, 61(2), 81-99.

Tsing, A. L. (2015). The mushroom at the end of the world: On the possibility of life in capitalist ruins. Princeton University Press.

Tsing, A. L., Bubandt, N., Gan, E. \& Swanson, H. A. (eds.). (2017). Arts of living on a damaged planet: Ghosts and monsters of the Anthropocene. University of Minnesota Press.

Van Dooren, T. (2014). Flight ways: Life and loss at the edge of extinction. Columbia University Press. 